\title{
Coupling impedance of a single hole in a thick-wall beam pipe
}

\author{
Robert L. Gluckstern \\ Physics Department, University of Maryland, College Park, Maryland 20742
}

(Received 26 February 1992)

\begin{abstract}
The coupling impedance of a hole in a thick-wall beam pipe has been calculated for hole dimensions that are small compared to the wavelength. In particular, the longitudinal and transverse coupling impedances are shown to be proportional to the difference between the transverse magnetic susceptibility and the electric polarizability of the inside surface of the hole, and these quantities are themselves proportional to the cube of the hole dimensions.
\end{abstract}

PACS number(s): 41.20.-q, 03.40.Kf, 03.50.De, 41.75.-i

\section{INTRODUCTION}

The calculation of the coupling impedance of a small hole in the wall of a beam pipe, at frequencies near or above the cutoff of the pipe, is of current interest. In particular, a liner with holes or slots is being considered to remove the heat generated by synchrotron radiation in the high current rings being designed at the superconducting supercollider (SSC) [1] and at the CERN Large Hadron Collider (LHC) [2]. In addition, a slotted cylindrical tube is being considered as a compact wiggler for free-electron lasers (FEL's) [3, 4].

Kurennoy [5] has calculated the longitudinal and transverse coupling impedance of a hole or slot in a thin metallic beam pipe wall. In the present calculation we shall use a more direct method than Kurennoy and also obtain the results for a hole or slot in a wall whose thickness may be comparable with the dimensions of the hole.

\section{LONGITUDINAL COUPLING IMPEDANCE}

We consider a point charge $Q$ traveling along the axis of a circular waveguide of radius $b$ at extreme relativistic velocity $v \cong c$. The definition of the frequency-dependent longitudinal coupling impedance of any obstacle can be taken to be

$$
Z_{\|}(k)=-\frac{1}{Q} \int_{-\infty}^{\infty} d z e^{j k z} E_{z}(0, \theta, z ; k)
$$

where $E_{z}(r, \theta, z ; k)$ is the axial electric field in the frequency domain, with frequency dependence $\exp (j \omega t)$, where $\omega=k c$.

The field component $E_{z}(r, \theta, z ; k)$ can be written as

$E_{z}(r, \theta, z ; k)=\sum_{n} \int_{-\infty}^{\infty} d q e^{-j q z-j n \theta} A_{n}(q) \frac{J_{n}(K r)}{J_{n}(K b)}$

which is the general solution of the wave equation which is regular at $r=0$. Here $K$, defined by

$$
K^{2}=k^{2}-q^{2},
$$

is the radial propagation constant and $A_{n}(q)$ are expansion coefficients to be determined by the geometry. The contour goes below any poles on the negative real $q$ axis and above any poles on the positive real $q$ axis in order to satisfy the outgoing wave boundary condition for the fields generated by the obstacle. The source fields in the frequency domain generated by the charge traveling at $v=c$ are

$E_{r}^{(s)}(r, \theta, z ; k)=Z_{0} H_{\theta}^{(s)}(r, \theta, z ; k)=\frac{Z_{0} Q}{2 \pi r} e^{-j k z}$

$E_{z}^{(s)}(r, \theta, z ; k)=0$

where $Z_{0}=120 \pi \Omega$.

If we set $r=0$ in Eq. (2.2), only the $n=0$ term survives, and Eq. (2.1) becomes

$$
\begin{aligned}
Z_{\|}(k) & =-\frac{1}{Q} \int_{-\infty}^{\infty} \frac{d q A_{0}(q)}{J_{0}(K b)} \int_{-\infty}^{\infty} d z e^{-j z(q-k)} \\
& =-\frac{2 \pi}{Q} A_{0}(k),
\end{aligned}
$$

where the last form is obtained because

$$
\int_{-\infty}^{\infty} d z e^{-j z(q-k)}=2 \pi \delta(q-k)
$$

For an obstacle configuration which does not extend into the pipe $(r<b)$, one can perform a Fourier inversion of Eq. (2.2) in $z$ and $\theta$ for $r=b$ to obtain

$A_{n}(q)=\frac{1}{4 \pi^{2}} \int_{0}^{2 \pi} d \theta \int_{-\infty}^{\infty} d z e^{j k z+j n \theta} E_{z}(b, \theta, z ; k)$.

Thus the longitudinal coupling impedance in Eq. (2.5) can be written as

$$
Z_{\|}(k)=-\frac{1}{2 \pi b Q} \iint d S e^{j k z} E_{z}(b, \theta, z ; k),
$$

where the integral extends only over the inner surface of any openings in the beam pipe, such as a hole. The task is to obtain $E_{z}$ from the source fields.

We now assume that the hole is small enough that the field components $E_{r}$ and $H_{\theta}$ in the beam pipe are well approximated by Eq. (2.4). Furthermore, we use the quasistatic solutions for the field components in the 
vicinity of the hole for hole dimensions small compared to the wavelength $2 \pi / k$. With these approximations one can express the surface integral as [6]

$$
\iint d S e^{j k z} E_{z}(b, \theta, z ; k)=j k\left(p_{r}-Z_{0} m_{\theta}\right)
$$

where $p_{r}$ and $m_{\theta}$ are the induced electric and magnetic dipole moments at the inner surface of the hole. These are induced by the values of $E_{r}$ and $H_{\theta}$ existing in the absence of the hole, and can be written as

$$
\begin{aligned}
& p_{r}=\frac{Z_{0} Q}{4 \pi b}\left(\chi_{s}+\chi_{a}\right) \\
& Z_{0} m_{\theta}=\frac{Z_{0} Q}{4 \pi b}\left(\psi_{s}+\psi_{a}\right)_{\theta}
\end{aligned}
$$

where $\psi_{s}, \psi_{a}, \chi_{s}, \chi_{a}$ are the symmetric and antisymmetric susceptibility (in the $\theta$ direction) and polarizability of the hole. (The $s$ and $a$ subscripts relate to the solutions of the symmetric and asymmetric potential problems in the vicinity of the hole [6].) Thus,

$$
\frac{Z_{\|}(k)}{Z_{0}}=\frac{j k}{8 \pi^{2} b^{2}}\left(\psi_{\mathrm{in}}-\chi_{\mathrm{in}}\right)
$$

where $\psi_{\text {in }}=\psi_{s}+\psi_{a}$ and $\chi_{\text {in }}=\chi_{s}+\chi_{a}$ are the inside susceptibility and polarizability of the hole. For a circular hole of radius $a$ in a wall of zero thickness

$$
\begin{aligned}
& \psi_{s}=\frac{8}{3} a^{3}, \psi_{a}=0, \\
& \chi_{s}=\frac{4}{3} a^{3}, \chi_{a}=0,
\end{aligned}
$$

so that

$$
\frac{Z_{\|}(k)}{Z_{0}}=\frac{j k a^{3}}{6 \pi^{2} b^{2}}(\text { circular hole in a thin wall }),
$$

a result which agrees with Kurennoy [5]. In the present calculation we have avoided the infinite-mode sums needed in his analysis and do not assume a vanishing wall thickness.

\section{TRANSVERSE COUPLING IMPEDANCE}

The calculation of the transverse coupling impedance proceeds in a similar manner. We start with the definition of the $x$ component of the transverse coupling impedance

$$
Z_{x}(k)=\frac{j}{Q \Delta_{x}} \int_{-\infty}^{\infty} d z e^{j k z}\left[E_{x}(z ; k)-Z_{0} H_{y}(z ; k)\right]_{\substack{x=0 \\ y=0}}
$$

in units of $\Omega / \mathrm{m}$ (of transverse displacement), where the source field comes from a test charge $Q$ traveling with $v \cong c$ at $x=\Delta_{x}, y=0$. The Panofsky-Wenzel theorem [7] corresponds to the simplification of Eq. (3.1) that comes about by using the Faraday law

$$
\frac{\partial E_{x}}{\partial z}-\frac{\partial E_{z}}{\partial x}=-j k Z_{0} H_{y}
$$

and leads to the alternate expression

$$
Z_{x}(k)=-\frac{1}{k Q \Delta_{x}} \int_{-\infty}^{\infty} d z e^{j k z}\left(\frac{\partial E_{z}}{\partial x}\right)_{\substack{x=0 \\ y=0}} .
$$

Once again we use the form in Eq. (2.2) for $E_{z}$, but this time we extract the $n= \pm 1$ terms, using $J_{1}(K r) \cong K r / 2$ for small $r$, to obtain

$$
\left(\frac{\partial E_{z}}{\partial x}\right)_{\substack{x=0 \\ y=0}}=\int_{-\infty}^{\infty} d q e^{-j q z} \frac{K\left[A_{1}(q)+A_{-1}(q)\right]}{2 J_{1}(K b)}
$$

where

$$
A_{n}(q)=\frac{1}{4 \pi^{2} b} \iint d S e^{j q z+j n \theta} E_{z}(b, \theta, z ; k) .
$$

The transverse coupling impedance can therefore be written as

$$
Z_{x}(k)=-\frac{2 \pi}{k Q \Delta_{x} b}\left[A_{1}(k)+A_{-1}(k)\right] .
$$

The source fields can be obtained as solutions of the transverse Laplace equation for the dipole term, multiplied by $\exp (-j k z)$. For this case we find

$$
\begin{aligned}
E_{r}^{(s)}(r, \theta, z ; k) & =Z_{0} H_{\theta}^{(s)}(r, \theta, z ; k) \\
& =Z_{0} \frac{Q \Delta_{x}}{2 \pi} \cos \theta\left(\frac{1}{r^{2}}+\frac{1}{b^{2}}\right) e^{-j k z}, \\
E_{z}^{(s)}(r, \theta, z ; k) & =0,
\end{aligned}
$$

and Eq. (3.5) yields

$$
A_{ \pm 1}(k)=\frac{-j k e^{ \pm j \theta} Z_{0} Q \Delta_{x} \cos \theta}{8 \pi^{3} b^{3}}\left(\psi_{\text {in }}-\chi_{\text {in }}\right) .
$$

Our result for the transverse impedance is therefore

$$
\frac{Z_{x}(k)}{Z_{0}}=\frac{j \cos ^{2} \theta}{2 \pi^{2} b^{4}}\left(\psi_{\text {in }}^{(\theta)}-\chi_{\text {in }}\right)
$$

For a circular hole of radius $a$ located at an azimuth $\theta$ in a wall of zero thickness, we have

$$
\frac{Z_{x}(k)}{Z_{0}}=\frac{j 2 a^{3} \cos ^{2} \theta}{3 \pi^{2} b^{4}}
$$

a factor of 2 smaller than Kurennoy [5]. We believe that he has incorrectly eliminated the term involving the electric polarizability.

\section{EFFECT OF SLOT SHAPE AND FINITE WALL THICKNESS}

For a zero thickness wall, analytic results are available [8] for an elliptical hole. In this case one has the relation

$$
\chi=\frac{\psi \zeta}{\psi+\zeta},
$$

where $\zeta$ is the susceptibility in the axial direction. Thus 
TABLE I. Polarizability and susceptibility of a circular hole in a thick wall.

\begin{tabular}{cccc}
\hline \hline$t / a$ & $3 \psi_{\text {in }} / 8 a^{3}$ & $3 \chi_{\text {in }} / 8 a^{3}$ & $3\left(\psi_{\text {in }}-\chi_{\text {in }}\right) / 4 a^{3}$ \\
\hline 0 & 1.000 & 0.500 & 1.000 \\
0.1 & 0.871 & 0.459 & 0.824 \\
0.3 & 0.778 & 0.438 & 0.680 \\
0.6 & 0.732 & 0.431 & 0.602 \\
1.0 & 0.715 & 0.430 & 0.570 \\
$\geq 2.0$ & 0.710 & 0.429 & 0.562 \\
\hline \hline
\end{tabular}

$$
\psi-\chi=\frac{\psi^{2}}{\psi+\zeta}
$$

for a hole where the major axis is parallel to $z$. If the semimajor and minor axes are $a_{z}$ and $a_{\theta}$, and if the ellipse is elongated such that $a_{z} \gg a_{\theta}$, then

$$
\psi \cong \frac{2 \pi}{3} a_{z} a_{\theta}^{2}, \quad \zeta \cong \frac{2 \pi a_{z}^{3} / 3}{\left[\ln \left(4 a_{z} / a_{\theta}\right)-1\right]} \gg \psi,
$$

and the longitudinal and transverse impedances will be further reduced to

$$
\begin{aligned}
& \frac{Z_{\|}(k)}{Z_{0}}=\frac{j k}{9 \pi b^{2}} \frac{a_{\theta}^{4}}{a_{z}}\left[\ln \frac{4 a_{z}}{a_{\theta}}-1\right], \\
& \frac{Z_{x}(k)}{Z_{0}} \cong \frac{j \cos ^{2} \theta}{3 \pi b^{4}} \frac{a_{\theta}^{4}}{a_{z}}\left[\ln \frac{4 a_{z}}{a_{\theta}}-1\right] .
\end{aligned}
$$

The polarizability and susceptibilities of a rectangular slot do not satisfy Eq. (4.1) exactly so the estimate of the reduction in Eq. (4.4) is only approximate. Nor is Eq. (4.1) satisfied for a circular or any other shaped slot in a thick wall, but a similar reduction is anticipated.

Finally, accurate values of the "inside" polarizability and susceptibility for a circular hole of radius $a$ in a wall of thickness $t$ can be calculated from a variational formulation for these quantities [6]. Table I for selected values of $t / a$ is given below. The coupling impedance falls quickly with wall thickness, reaching a value for an infinite thickness which is $56 \%$ of its value for zero thickness.

\section{MEASUREMENT OF POLARIZABILITY AND SUSCEPTIBILITY OF A HOLE}

We denote the fields in the absence of the hole as $\mathbf{E}_{1}, \mathbf{H}_{1}$. We neglect radiation through the hole and take $\mathbf{H}$ to be $90^{\circ}$ out of phase with $\mathbf{E}$ in time, and use $\mathbf{G}_{1}=Z_{0} \mathbf{H}_{1}, \mathbf{G}_{2}=Z_{0} \mathbf{H}_{2}$, so that $\mathbf{E}$ and $\mathbf{G}$ have the same units. The corresponding resonant frequencies are $\omega_{1}=k_{1} c, \omega_{2}=k_{2} c$.

Starting with $\int d v \boldsymbol{\nabla} \cdot\left(\mathbf{E}_{2} \times \mathbf{G}_{1}-\mathbf{E}_{1} \times \mathbf{G}_{2}\right)$ one can obtain a simple formula for the frequency change $k_{2}-k_{1}$,

$$
k_{2}-k_{1}=\frac{\int d S \mathbf{n} \cdot \mathbf{E}_{2} \times \mathbf{G}_{1}}{\int d v \mathbf{E}_{1} \cdot \mathbf{E}_{2}+\int d v \mathbf{G}_{1} \cdot \mathbf{G}_{2}}
$$

where the surface integral is over the inner wall surface of the hole. For a small hole one may approximate $\mathbf{E}_{2}$ and $\mathbf{G}_{2}$ by $\mathbf{E}_{1}$ and $\mathbf{G}_{1}$ in the denominator, which then is directly related to the stored energy in the cavity. At the same time, the numerator is closely related to surface integral considered in Eq. (2.9) and expressed in Eq. (2.10) in terms of the inside polarizability and susceptibility of the hole. Specifically, using a rectangular coordinate system $(x, y, z)$ at the hole with $z$ being perpendicular to the wall, we have

$$
\int d S \mathbf{n} \cdot \mathbf{E}_{2} \times \mathbf{G}_{1}=\iint d x d y\left[E_{2 x} G_{1 y}-E_{2 y} G_{1 x}\right] .
$$

The technique for evaluating such integrals is detailed elsewhere [9]. In essence, if we use the static approximation for $\mathbf{E}_{2}$ and $\mathbf{G}_{1}$, Eq. (5.2) vanishes. A first-order Taylor expansion of $\mathbf{G}_{1}$ in the vicinity of the hole and a static approximation to $\mathbf{E}_{2}$ leads to the electric dipole moment contribution. A static approximation to $\mathbf{G}_{1}$ and use of the Faraday law $\boldsymbol{\nabla} \times \mathbf{E}_{2}=k_{2} \mathbf{G}_{2}$ leads to the magnetic dipole contribution. After some algebra, one finds for the detuning of the cavity

$\frac{k_{2}-k_{1}}{k_{1}} \cong \frac{\frac{1}{4}\left(E_{z}^{2} \chi_{\mathrm{in}}-Z_{0}^{2} H_{x}^{2} \psi_{\mathrm{in}}^{x}-Z_{0}^{2} H_{y}^{2} \psi_{\mathrm{in}}^{y}\right)}{\int E^{2} d v=Z_{0}^{2} \int H^{2} d v}$,

where $x$ and $y$ are chosen along the principal axes of the inside hole susceptibility. The field components $E_{z}, H_{x}, H_{y}$ are those present in the absence of the hole.

It is therefore a simple matter to measure the inside polarizability and susceptibility of the hole by measuring the detuning of the cavity caused by the hole. In fact, one can also measure the "outside" susceptibility and polarizability $\left(\psi_{\text {out }} \equiv \psi_{s}-\psi_{a}, \chi_{\text {out }} \equiv \chi_{s}-\chi_{a}\right)$ by determining the fields induced in an adjacent cavity tuned to a different frequency.

\section{SUMMARY}

We have obtained expressions for the longitudinal and transverse coupling impedance of a hole in a beam pipe in terms of the inside polarizability and susceptibility of the hole. For a hole of given cross-sectional area, the impedance can be reduced by elongating the hole parallel to the axis of the pipe. The modification due to the wall thickness is tabulated for a circular hole.

The inside polarizability and susceptibility can be easily measured by determining the change of resonant frequency caused by a hole of the same geometry in a cavity whose field solutions are calculable.

\section{ACKNOWLEDGMENTS}

This work was supported partly by the U.S. Department of Energy. The author would like to thank F. Caspers, G. Dôme, F. Ruggiero, and B. Zotter for helpful conversations. He is also grateful to SL Division at CERN for its hospitality during the course of this work. 
[1] A. Chao (private communication).

[2] F. Caspers, F. Ruggiero, and B. Zotter (private communication).

[3] R.W. Warren, Nucl. Instrum. Methods A 304, 765 (1991).

[4] R.L. Gluckstern, Phys. Rev. A 15, 44, 3889 (1991).

[5] S. Kurennoy, CERN Report No. SL/91-29 (AP), 1991.

[6] R.L. Gluckstern and J.A. Diamond, IEEE Trans. Mi- crowave Theory Tech. 39, 274 (1991).

[7] W.K.H. Panofsky and W.A. Wentzel, Rev. Sci. Instrum. 27, 967 (1956).

[8] R.E. Collin, Field Theory of Guided Waves (McGraw-Hill, New York, 1960).

[9] R.L. Gluckstern, R. Li, and R.K. Cooper, IEEE Trans. Microwave Theory Tech. 38, 186 (1990); 38, 1529 (1990). 